\title{
Through-package fat determination in commercial samples of mayonnaise and salad dressing using time-domain nuclear magnetic resonance spectroscopy and chemometrics
}

\author{
Fabíola Manhas Verbi Pereira ${ }^{\mathrm{a}, *}$, Ana Paula Rebellato ${ }^{\mathrm{b}}$, Juliana Azevedo Lima Pallone ${ }^{\mathrm{b}}$, \\ Luiz Alberto Colnago ${ }^{\mathrm{a}}$ \\ ${ }^{a}$ Embrapa Instrumentação, Rua Quinze de Novembro 1452, São Carlos, SP 13561-206, Brazil \\ ${ }^{\mathrm{b}}$ Departamento de Ciência de Alimentos, Faculdade de Engenharia de Alimentos, Universidade Estadual de Campinas (UNICAMP). Campinas, \\ SP 13083-962, Brazil
}

\section{A R T I C L E I N F O}

\section{Article history:}

Received 13 August 2013

Received in revised form

24 December 2013

Accepted 14 February 2014

Available online 28 February 2014

\section{Keywords:}

Food analysis

TD-NMR

Chemometrics

Fat content

Clean method

\begin{abstract}
A B S T R A C T
Although fat is an essential component in the human diet, the consumption of food with high fat content has been the major cause of obesity. Here we are demonstrating that time-domain nuclear magnetic resonance (TD-NMR) decays, obtained by Carr-Purcell-Meiboom-Gill (CPMG) pulse sequence, can be used to predict the total fat content in sealed packages of commercial food emulsions, such as mayonnaise and salad dressing that may contain more than $50 \%$ of fat. The PLS model of the CPMG decays shows high linear correlation $(>0.9)$ and low root mean square errors (RMSE) for cross-validation and validation data. Therefore, this procedure can be used to predict the total fat content in food emulsion after packaging, to detect problems in the manufacturing process or fraud. In addition, the TD-NMR instrument and the proposed methods can also be adapted for using at the end of the food chain (store) to measure the fat content in consumer packaged goods.
\end{abstract}

(C) 2014 Elsevier Ltd. All rights reserved.

\section{Introduction}

Although fat is an essential component in the human diet (Wendin et al., 1997), the excessive consumption of food with high fat content has been the major cause of obesity, one of the most serious human health problems in the modern developed world. Therefore, the fast precise determination of the fat content in raw and processed foods is an important analytical task (Mullie, Godderis, \& Clarys, 2012).

Comparing the analytical methods used in fat determination, the standard wet chemical methods are usually expensive, destructive, time-consuming and they generate toxic waste due to the use of organic solvents (Hubbard, Sheppard, Newkirk, Prosser, \& Osgood, 1977; Yang, Lin, \& Choong, 2002). Therefore, the use of fast spectroscopic methods based on time-domain NMR (TD-NMR) spectroscopy, which generates minimal or even no waste, has

\footnotetext{
* Corresponding author. Current address: Departamento de Química Analítica, Instituto de Química, Universidade Estadual Paulista "Júlio de Mesquita Filho" (Unesp), Rua Prof. Francisco Degni, 55, Araraquara, SP 14800-900, Brazil. Tel.: +55 163301 9609; fax: +55 1633222308 .

E-mail addresses: fabiola@iq.unesp.br, fmverbi@uol.com.br (F.M. Verbi Pereira).
}

gained importance in recent years. An important review dedicated to the use of TD-NMR to analyze food products was published in last decade (van Duynhoven, Voda, Witek, \& Van As, 2010).

When food products, such as chocolate, margarine, butter, seeds and nuts, have low water content, the total fat concentration can be determined by TD-NMR using the intensity of the free induction decay (FID) or the echo signals and univariate statistical analysis (Colnago, Azeredo, Marchi Netto, Andrade, \& Venâncio, 2011; Guthausen et al., 2004; van Duynhoven et al., 2010). However, when the food products, such as mayonnaise and salad dressings have high water content, these methods are useless to quantify the fat content. Therefore, more sophisticated TD-NMR methods and/or multivariate analysis have to be used (Pereira, Carvalho, Cabeça, \& Colnago, 2013; Pereira \& Colnago, 2012; Rahmatallah, Li, Seton, Gregory, \& Aspden, 2006).

These NMR methods can produce acceptable results using the difference in relaxation times or the molecular diffusion of oil and water, by using the Carr-Purcell-Meiboom-Gill (CPMG) decays, or the continuous wave free precession (CWFP) (Venâncio, Engelsberg, Azeredo, Alem, \& Colnago, 2005) or the pulsed field gradient stimulated echo (PFGSE) pulse sequences (van Duynhoven et al., 2010). 
Guthausen et al. (2004) used a single-sided NMR spectrometer to measure the fat content of oil/water emulsions in different samples, such as margarine, mayonnaise and coffee cream. Although the analyses can be performed in the package, singlesided NMR sensors have a low signal-to-noise ratio. Therefore, for an average signal to be obtained, the analysis time must be increased to approximately $5 \mathrm{~min}$. Another limitation of a singlesided sensor is that the analysis is not made at the bulk but is instead limited to a very small volume at approximately the $3 \mathrm{~mm}$ mark of the NMR sensor.

The measurement time in a conventional bench-top timedomain NMR spectrometer is lower (ca. $1 \mathrm{~min}$ ) than in a singlesided NMR spectrometer. However, to use a conventional benchtop time-domain spectrometer, the samples must be removed from their packages and placed in a NMR tube, thus increasing the total analysis time similar to that when using the single-sided NMR spectrometer (van Duynhoven et al., 2010).

Here, we show that low-field TD-NMR spectrometers based on a wide bore (10 $\mathrm{cm}$ probe) Halbach permanent magnet can be used to measure the fat content in packaged samples in approximately $20 \mathrm{~s}$ for each sample. Therefore, this NMR equipment combined with chemometric analysis can be a rapid inline or offline quality control and quality assurance method for determining the fat content in consumer packaged goods with high water contents, such as mayonnaise and salad dressings.

\section{Materials and methods}

\subsection{Samples}

All mayonnaise and dressing samples were purchased at local markets (São Carlos, São Paulo State, Brazil). A total of 31 mayonnaise samples from three world-renowned manufacturers were investigated, among them, 21 samples from the same lot were used to perform the multivariate models, and 10 samples from another lot were used to test the predictive ability of these models. The numbers of samples with varying fat contents according to the product labels

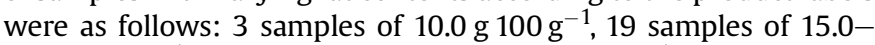

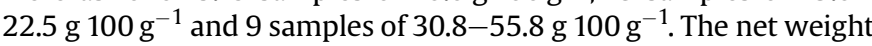
of the packages varied between 400 and $500 \mathrm{~g}$. Fifteen salad dress-

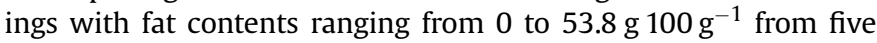
different manufacturers were also evaluated, being 3 samples of

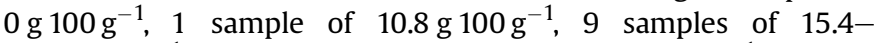

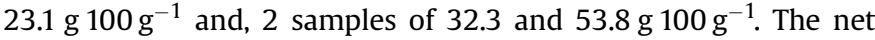
weight of salad dressings packets ranged from 234 to $245 \mathrm{~g}$.

\subsection{TD-NMR CPMG measurements}

The signal acquisitions from food emulsions (mayonnaise and salad dressing samples) through the sealed packages were performed using a NMR spectrometer (Spinlock Magnetic Resonance Solution, Cordoba, Argentina) with a permanent Halbach magnet of $0.23 \mathrm{~T}\left(9 \mathrm{MHz}\right.$ for $\left.{ }^{1} \mathrm{H}\right), 10 \mathrm{~cm}$ bore and $50 \mathrm{~cm}$ long analytical magnet, according to the diagram of Fig. 1. The CPMG sequence was performed with $\pi / 2$ and $\pi$ pulses of 9.2 and $18.08 \mu \mathrm{s}$, respectively; the echo time was $400 \mu \mathrm{s}$ ( $\tau=200 \mu \mathrm{s})$, and 2500 echoes were used. The samples were in thermal equilibrium with the NMR probe $\left(22^{\circ} \mathrm{C}\right)$. Each sample was analyzed in duplicate and on three different days, producing a total of 6 CPMG signals per sample.

\subsection{Reference values for total lipids}

Three replicates for each mayonnaise and salad dressing packet were performed using the Bligh and Dyer (1959) method for the extraction of lipids.

\subsection{Data investigation}

First, the TD-NMR CPMG signals were exploited using principal component analysis (PCA). The univariate model for fat content prediction was applied using the transverse relaxation time $\left(\mathrm{T}_{2}\right)$ values exponentially fitted to a function available in Origin 8.1 (OriginLab, Northampton, MA, USA). The chemometric technique partial least squares (PLS) was used to compute the multivariate model for fat content prediction. PCA and PLS are available in Pirouette 4.0 rev. 2 software (Infometrix, Bothell, USA). The ratio of number samples' for construction of the prediction models comprise a total of $80 \%$ of the data set were used for calibration and remaining $20 \%$ for validation.

\section{Results and discussion}

Fig. 2 shows the normalized CPMG decays of two samples of mayonnaise and two samples of salad dressing in sealed packages

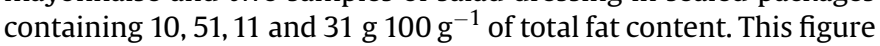
shows that, according to the CPMG time constant decays, the transverse relaxation time of the salad dressing $\left(T_{2}=0.4 \mathrm{~s}\right)$ with $11 \%$ fat is considerably longer than the relaxation time $\left(T_{2}=0.2 \mathrm{~s}\right)$ of the mayonnaise sample with a similar fat content (10\%). This difference reflects the variations in the chemical composition and the droplet size of the two emulsions.

However, when the mayonnaise and salad dressing samples were compared as two distinct foods products, $T_{2}$ showed an inverse correlation to the fat content. The mayonnaise and salad dressing samples with the higher fat contents (51 and 31\%) have smaller relaxation times $\left(T_{2}=0.1 \mathrm{~s}\right.$ and $\left.0.2 \mathrm{~s}\right)$ than the products with the lower fat contents. Therefore, the mayonnaise and salad dressing emulsions must be modeled as two distinct classes.

The analyses of NMR data were performed with the average of 6 CPMG signals for each sample, acquired in duplicate for 3 days for univariate and multivariate models. For instance, in the case of mayonnaise, the mean \pm standard deviation (SD) among the $T_{2}(\mathrm{~s})$ values computed for TD-NMR CPMG signals of the 6 replicates were $0.2 \pm 0.003$ (for the lowest fat content) and $0.1 \pm 0.003$ (for the highest fat content). In the case of salad dressing the values were in (s) $0.4 \pm 0.004$ (for the lowest fat content) and $0.2 \pm 0.003$ (for the highest fat content).

The mayonnaise univariate model was computed using the discrete $T_{2}$ values obtained by fitting the CPMG decays with monoand biexponential functions. The linear correlation coefficients $(r)$ using the monoexponential function fitting values were 0.70 and 0.85 for the training ( 15 samples) and the validation ( 6 samples) data sets, respectively. The low value $(r=0.48)$ of this same parameter for the fat content prediction of the 10 external samples shows that using the CPMG decays with a monoexponential fit was not a sufficiently accurate model. The biexponential values were also tested, and the correlation was lower $(r<0.4)$ than when using the monoexponential fit.

As previously demonstrated, better correlations have been obtained between CPMG multi-exponential decays and the chemical components by using multivariate analysis, which does not use discrete $T_{2}$ values but rather uses the full decay signals (Pereira et al., 2013; Pereira \& Colnago, 2012).

To apply the models, the reference values for the dependent variable (Y matrix) were determined using the lipid extraction method developed by Bligh and Dyer (1959). The label values of total fat content for the mayonnaise samples had a high linear correlation coefficient of 0.99 compared with those values obtained

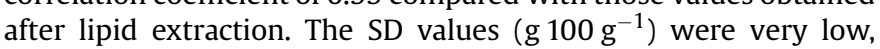
between 0.04 and 0.9 , and the relative $\mathrm{SD}(\%)$ was from 0.2 to 3.0. 


\section{Food packet inside of TD-NMR spectrometer}

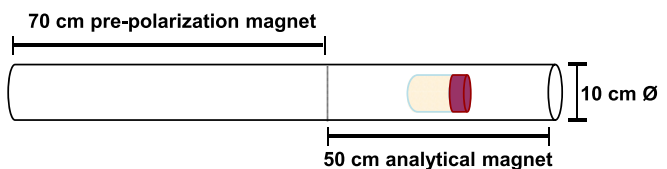

\section{PLS model for mayonnaises}

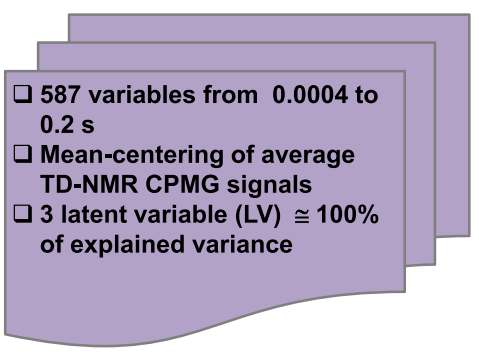

\section{PLS model for salad dressings}

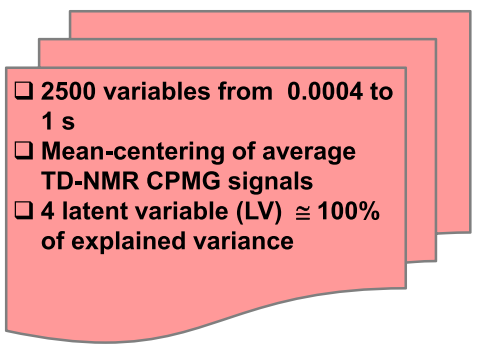

Data matrices for Chemometrics evaluation

Independent variables ( $X$ matrix)

2500 variables on columns (from 0.0004 to $1 \mathrm{~s}$ )

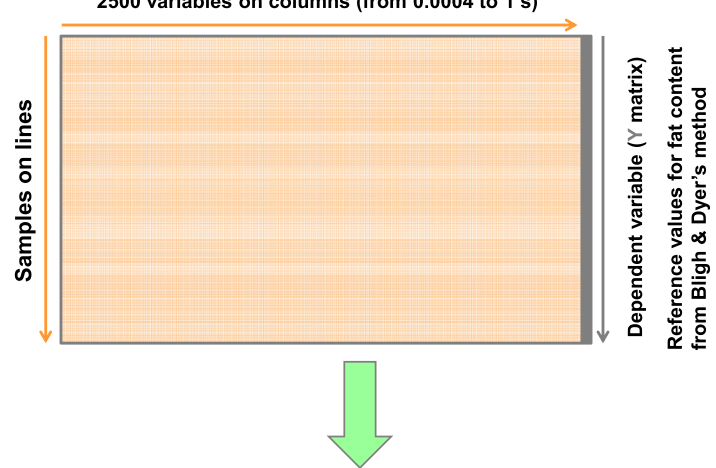

Variables Selection

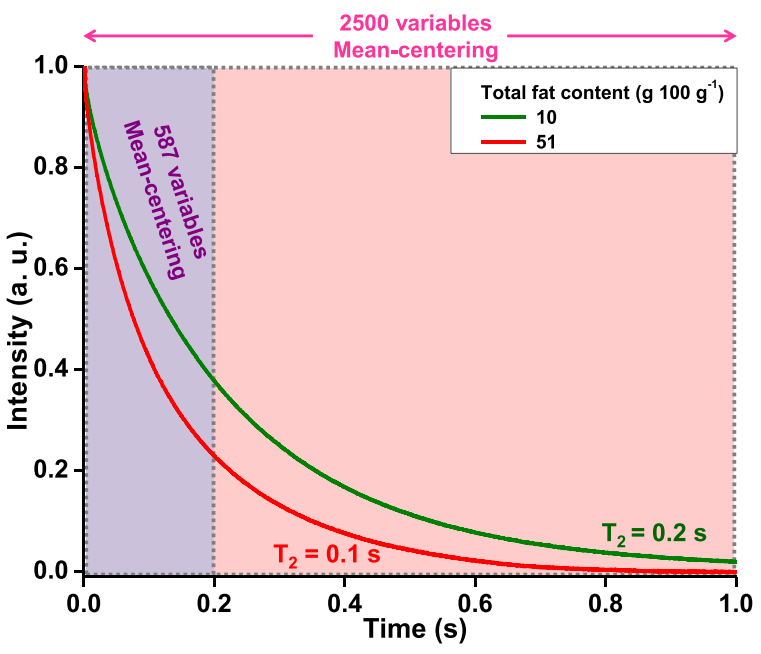

Fig. 1. General description of analytical method using TD-NMR CPMG signals and chemometrics.

It is important to emphasize the difficulty in determining the total fat content in emulsion samples, especially in salad dressing samples. In some cases, the tests must be repeated many times due to the repeatability limitations of Bligh and Dyer's method for this

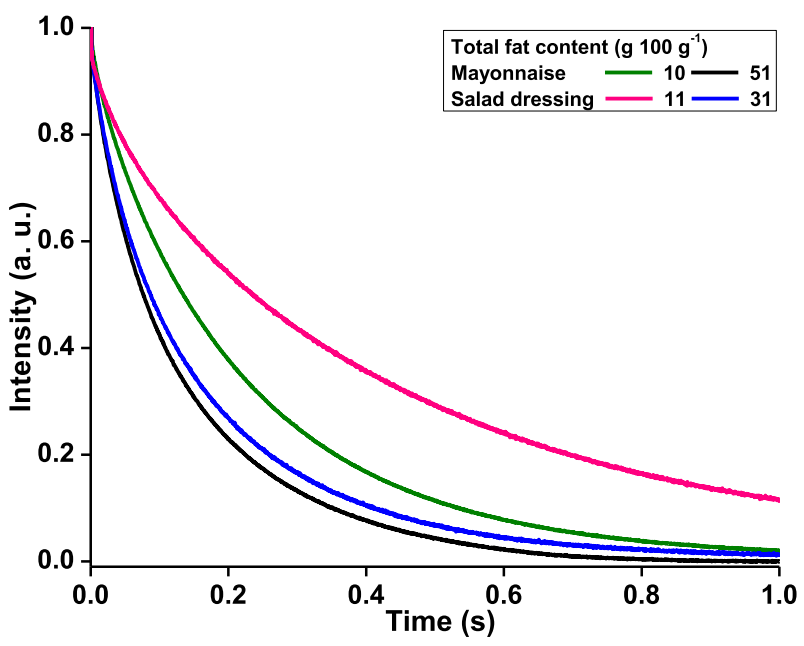

Fig. 2. Carr-Purcell-Meiboom-Gill (CPMG) decays of commercial mayonnaise and

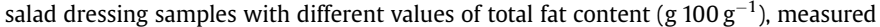
in sealed containers, using a $10 \mathrm{~cm}$ bore and $0.23 \mathrm{~T}$ Spinlock time-domain nuclear magnetic resonance (TD-NMR) spectrometer. type of emulsion. To overcome this challenge, measuring the entire and intact food package is desired.

Next, the multivariate PLS model was initially examined using full CPMG signals that included 2500 independent variables; in other words, the signals for the time from 0.0004 to $1 \mathrm{~s}$. For the calculations, the average decays were also mean-centered. The training and the validation data sets were composed by the same 16 and 5 samples previously tested for univariate model, respectively.

The best preprocessing procedure applied to the signals was mean-centering. In this case, auto-scaling of the independent variables ( $\mathbf{X}$ matrix) was not adequate because of the spectroscopy nature of the data. The profiles of the signals represent mostly short decays according to the fat content in the samples under analysis. Therefore, all the variables of the $\mathbf{X}$ matrix cannot have the same numerical level by scaling to the unit variance (auto-scaling).

Three latent variables (LV) were required to achieve the best predictions, which explained almost $100 \%$ of the variance in the data. The results of the regression vector indicated that the highest values were in the range of the first 587 variables, from 0.0004 to $0.2 \mathrm{~s}$. The same information was observed from the correlation spectrum. The residual values for the independent variables were very low, between -0.01 and $0.02 \mathrm{~s}$.

The choice for the optimal number of LVs of all PLS models presented in this investigation was based on the compromise between the least number of LVs that can minimize the root mean 
square error of cross-validation (RMSECV), and maximize the explained variance for the data.

Then, the PLS model for mayonnaise samples was performed using the range of the first 587 variables, from 0.0004 to $0.2 \mathrm{~s}$ and 3 LV. A very good linear fit was confirmed between the reference values and those predicted by PLS, with linear correlation coefficient values of 0.93 and 0.97 for the training (Fig. 3a) and the validation (Fig. 3b) data sets, respectively. The total fat contents in the 10 remaining samples from another lot were also successfully predicted by PLS with a high linear correlation coefficient of 0.93 (Fig. 3c). For the PLS model, these high $r$ values also confirm that this multivariate model can better predict the fat content in emulsions than the univariate model.

The results from using the PLS model shown in Fig. 3 indicate a potential for industrial application in food analysis considering the high linearity of the method for the investigated analytical range and that the signals were obtained from the entire contents of a mayonnaise package. In comparison, lipid extraction used the bulk material.

The important evaluated parameters, such as the RMSECV and the RMSE for validation (RMSEV), had values of 4.8 and

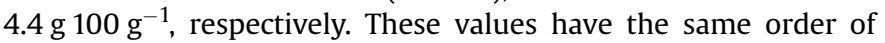
magnitude, which is strong evidence that the models are very promising for the prediction of fat contents in sealed packages of mayonnaise in industrial quality control for the consumer's care.

The potential of TD-NMR spectroscopy combined with PLS was also investigated for use on salad dressing samples, and it accurately predicted the fat content in those samples, as shown by the data in Fig. 4. For the products showing on their labels 0 content for the total fat; in fact, according to the Bligh and Dyer's method these

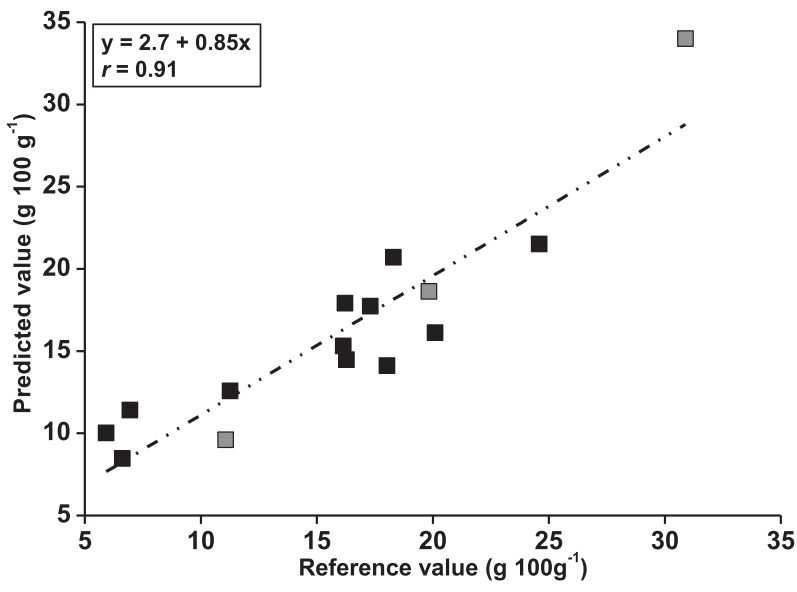

Fig. 4. Linear correlation between the predicted values from the partial least squares (PLS) model with the TD-NMR CPMG signals and those determined using Bligh and Dyer's method for commercial salad dressing samples.

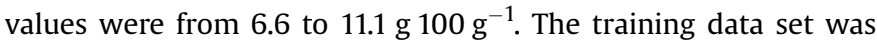
comprised of 12 samples and the 3 remaining samples were included in the validation data set. The chosen samples for the validation data set are noted by gray square symbols in the plot of Fig. 4. The linear coefficients computed for both separate sets, training and validation data were higher than 0.9 .

Four LV and the entire signal with 2500 variables were used to perform the modeling. The explained variance for $4 \mathrm{LV}$ was almost

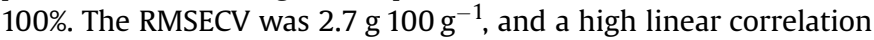
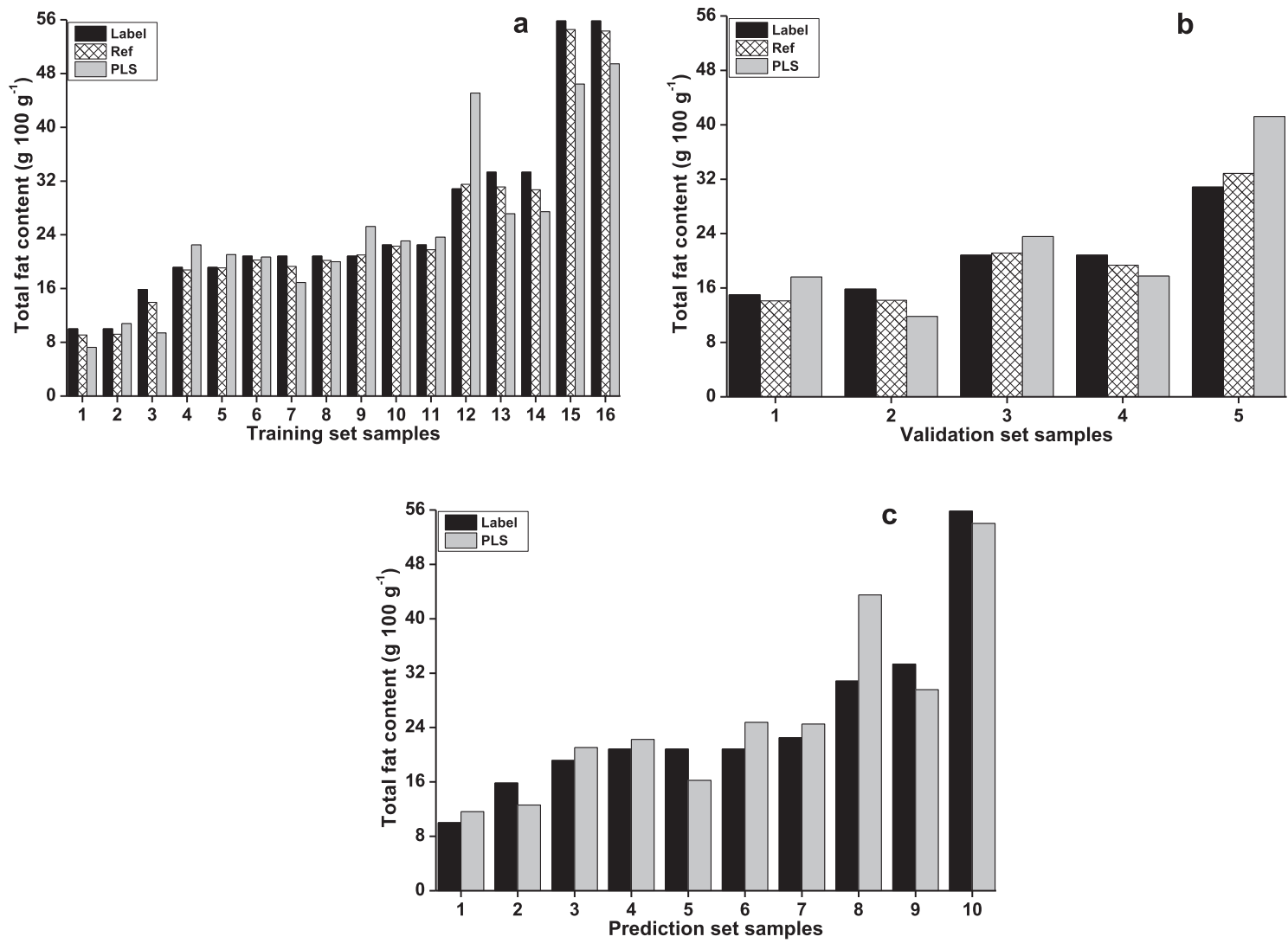

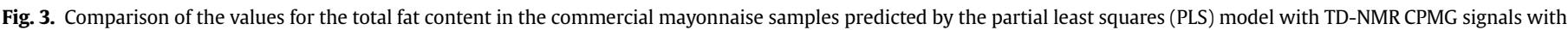

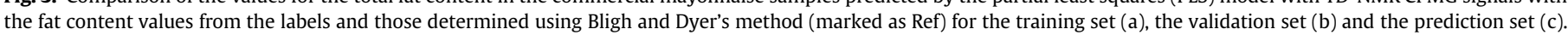


of 0.91 was observed between the values obtained from the trials using the classical method (Bligh and Dyer lipid extraction), confirming the applicability of the method proposed in this study for investigating the fat content in different types of food emulsions.

The most common packaging material for the mayonnaises and salad dressing is made of plastic. Then, for this investigation plastic packets were evaluated and they did not interfere or cause a high signal-to-noise ratio from the samples. However, this method cannot be used when the food is packaged in metallic materials, including plastic tubes, with aluminum foil layer. The metal strongly attenuates the radiofrequency preventing both the transmission and detection of the NMR signal.

\section{Conclusions}

The TD-NMR CPMG relaxation decays using PLS models successfully predicted the total fat content of mayonnaise and salad dressing samples, which had widely varying fat contents. TD-NMR instrument combined to Chemometrics model proposed here can be used for industrial quality control to measure the fat content in consumer packaged goods. In addition, this analytical method can be used to detect problems in the manufacturing process after packaging has occurred for the consumer's care.

\section{Acknowledgments}

We are grateful to the Conselho Nacional de Desenvolvimento Científico e Tecnológico (CNPq) for the fellowship awarded to F.M.V. Pereira (Process 150776/2012-8), CNPq grants (301087/2009-1 and 476819) and FAPESP grant (2012/20247-8).

\section{References}

Bligh, E. G., \& Dyer, W. J. (1959). A rapid method of total lipid extraction and purification. Canadian Journal of Biochemistry and Physiology, 37, 911-917.

Colnago, L. A., Azeredo, R. B. V., Marchi Netto, A., Andrade, F. D., \& Venâncio, T. (2011). Rapid analyses of oil and fat content in agri-food products using continuous wave free precession time domain NMR. Magnetic Resonance in Chemistry, 49, S113-S120.

Guthausen, A., Guthausen, G., Kamlowski, A., Todt, H., Burk, W., \& Schmalbein, D. (2004). Measurement of fat content of food with single-sided NMR. Journal of the American Oil Chemists' Society, 81, 727-731.

Hubbard, W. D., Sheppard, A. J., Newkirk, D. R., Prosser, A. R., \& Osgood, T. (1977). Comparison of various methods for the extraction of total lipids, fatty acids, cholesterol, and other sterols from food products. Journal of the American Oil Chemists' Society, 54, 81-83.

Mullie, P., Godderis, L., \& Clarys, P. (2012). Determinants and nutritional implications associated with low-fat food consumption. Appetite, 58, 34-38.

Pereira, F. M. V., Carvalho, A. S., Cabeç, L. F., \& Colnago, L. A. (2013). Classification of intact fresh plums according to sweetness using time-domain nuclear magnetic resonance and Chemometrics. Microchemical Journal, 108, 14-17.

Pereira, F. M. V., \& Colnago, L. A. (2012). Determination of the Moisture Content in Beef Without Weighing Using Benchtop Time-Domain Nuclear Magnetic Resonance Spectrometer and Chemometrics. Food Analytical Methods, 5, 13491353.

Rahmatallah, S., Li, Y., Seton, H. C., Gregory, J. S., \& Aspden, R. M. (2006). Measurement of relaxation times in foodstuffs using a one-sided portable magnetic resonance probe. European Food Research E Technoogy, 222, 298-301.

van Duynhoven, J., Voda, A., Witek, M., \& Van As, H. (2010). Time-domain NMR applied to food products. Annual Reports on NMR Spectroscopy, 69, 145-197.

Venâncio, T., Engelsberg, M., Azeredo, R. B. V.,N., Alem, A. E., \& Colnago, L. A. (2005). Fast and simultaneous measurement of longitudinal and transverse NMR relaxation times in a single continuous wave free precession experiment. Journal of Magnetic Resonance, 173, 34-39.

Wendin, K., Aaby, K., Edris, A., Ellekjaer, M. R., Albin, R., Bergenståhl, B., \& et al. (1997). Low-fat mayonnaise: influences of fat content, aroma compounds and thickeners. Food Hydrocolloids, 11, 87-99.

Yang, M.-H., Lin, H.-J., \& Choong, Y.-M. (2002). A rapid gas chromatographic method for direct determination of BHA, BHT and TBHQ in edible oils and fats. Food Research International, 35, 627-633. 